\title{
An unusual case of asymmetrical combined variations of the subclavian and axillary artery with clinical significance
}

\author{
E. Panagouli ${ }^{1}$, K. Natsis ${ }^{2 *}$, M. Piagkou ${ }^{1 *}$, G. Kostare1, G. Tsoucalas ${ }^{3}$, D. Venieratos ${ }^{1}$ \\ ${ }^{1}$ Department of Anatomy, Medical School, Faculty of Health Sciences, \\ National and Kapodistrian University of Athens, Greece \\ 2Department of Anatomy and Surgical Anatomy, Faculty of Health Sciences, Aristotle University of Thessaloniki, Greece \\ ${ }^{3}$ Department of Anatomy, Medical School, Democritus University of Thrace, Alexandroupolis, Greece
}

[Received: 20 December 2018; Accepted: 9 February 2019]

\begin{abstract}
In a Greek Caucasian male cadaver, a combination of the following arterial variations were observed: an aberrant right subclavian artery originating as a last branch of the aortic arch and coursed posterior to the oesophagus, a right non-recurrent laryngeal nerve, an atypical origin of the left suprascapular artery from the axillary artery, an unusual emersion of the lateral thoracic artery from the subscapular artery and a separate origin of the left thoracodorsal artery from the axillary artery. According to the available literature the corresponding incidences of the referred variants are: $0.7 \%$ for the aberrant right subclavian artery, $1.6-3.8 \%$ for the origin of the suprascapular artery from the axillary artery, 3\% for the origin of the left thoracodorsal artery from the axillary artery and $30 \%$ for the origin of the lateral thoracic artery from the subscapular artery. Such unusual coexistence of arterial variations may developmentally be explained and has important clinical significance. (Folia Morphol 2019; 78, 4: 883-887)
\end{abstract}

Key words: aberrant right subclavian artery, suprascapular artery, lateral thoracic artery, thoracodorsal artery, subscapular artery, oesophagus, non-recurrent laryngeal nerve

\section{INTRODUCTION}

According to the classical anatomical textbooks, the aortic arch (AOA) is the continuation of the ascending aorta at the level of the upper border of the $2^{\text {nd }}$ right sternocostal joint. The $A O A$, at its lateral aspect, gives off the brachiocephalic trunk (BCT), the left common carotid artery (LCCA) and the left subclavian artery (LSCA) [20]. Their point of origin, as well as the distance between them vary [20]. The right subclavian artery (RSCA) and the right common carotid artery (RCCA) arise from the $B C T$, while the LSCA usually originates independently from the AOA after the LCCA origin [20].

Several variations of the AOA branching pattern have been reported [10, 20]. Primary branches may be reduced to $2(13 \%)$ or rarely to 1 (less than $0.1 \%$ ) [10]. An additional branch, more commonly the vertebral artery (VA), may be present at $4 \%$ of the cases [10]. The LCCA may originate from the BCT with an incidence ranging between $7 \%$ and $13 \%$. More rarely in less than $1 \%$, the RCCA and RSCA may arise separately, with the latter branching as a last branch of the AOA and coursing posterior to the oesophagus $[10,20]$. When this variant occurs, the right inferior laryngeal nerve hooks round the RCCA and is characterised as non-recurrent laryngeal nerve [20].

The suprascapular artery (SSA) originates from the thyrocervical trunk (TCT) of the subclavian artery, although it may arise from the $3^{\text {rd }}$ part of the sub- 
clavian artery [20]. Rarely (less than 1\%), it may be a branch of the axillary artery (AA) [10].

The $A A$ is the subclavian artery continuation from the lateral margin of the $1^{\text {st }}$ rib and supplies the axillary region [20]. The pectoralis minor muscle divides the AA into three parts: the $1^{\text {st }}$ part gives rise to the superior thoracic artery, the $2^{\text {nd }}$ part to the thoracoacromial trunk (TAT) and lateral thoracic artery (LTA) and the $3^{\text {rd }}$ part to the subscapular artery (SBSA) and the anterior and posterior circumflex humeral arteries [20]. The AA presents a great variability from its typical pattern [10], as it tends to form common trunks in about $50 \%$ of the cases, while accessory main (profunda brachii artery) and muscular branches seem to arise from the AA in approximately $20 \%$ of the cases $[10,22]$.

In the present study, an aberrant right subclavian artery (ARSCA) originating as a last branch of the AOA and coursed posterior to the oesophagus coexisted with a right non-recurrent laryngeal nerve (RNRLN), with an atypical origin of the LSCA from the AOA, an unusual branching of the LTA from the SBSA and a separate origin of the left thoracodorsal artery (TDA) from the $A A$.

\section{CASE REPORT}

The reported anatomical variations were discovered in the upper extremities of a Greek Caucasian male cadaver (deceased at the age of 78 years), during routine educational dissection at the Department of Anatomy of the Medical School of National and Kapodistrian University of Athens. The cadaver derived from the body donation programme after a signed informed consent by the donator himself.

While dissecting the structures at the right side of the neck, the RCCA, the internal jugular vein and the vagus nerve $(X)$ were identified. The RSCA and the RCCA were identified and the BCT absence was noticed (Fig 1A). The RCCA originated from the median aspect of the AOA, followed by the LCCA and LSCA. In continuation, the ARSCA originated from the posterolateral aspect of the AOA and coursed posterior to the oesophagus before entering the supraclavicular space (Fig 1A). Additionally, an aneurysm of $32.3 \mathrm{~mm}$ was found at the point of the ARSCA emersion. Following the dissection, a branch of the right vagus nerve was given off in the lower rim of the thyroid cartilage. The atypical branch coursed through the thyroid gland, instead of coursing around the RSCA and then upwards before en- tering the thyroid gland. Therefore the branch was considered as a RNRLN (Fig 1B).

The retro-oesophageal ARSCA (length of $14.5 \mathrm{~cm}$ ) continued its anomalous course and gave rise to the right VA ( $2 \mathrm{~mm}$ of diameter) and the right thyrocervical trunk which arose from the same point of origin with the internal thoracic artery (Fig 1B). The right VA presented an important stenosis at its point of origin. The ARSCA continued its course as the right $A A$ ( $7.28 \mathrm{~mm}$ of diameter) with no further variations (Fig 1B).

The LSCA (6.94 $\mathrm{mm}$ of diameter) had an unexpected branching pattern. The left VA and the costocervical trunk presented no variations. The left SSA originated from the $1^{\text {st }}$ part of the $A A, 5.2 \mathrm{~cm}$ after the thyrocervical trunk origin, by a common stem with two branches that supplied the upper surface of the subscapularis muscle (Fig 1C). After a course of $2.6 \mathrm{~cm}$, the left AA (5.09 $\mathrm{mm}$ of diameter) gave rise to the TAT and $3.7 \mathrm{~cm}$ below the TAT emerged the SBSA which typically gave rise to the circumflex scapular artery. Then a large trunk was observed, continuing downwards and gave four branches, three for the serratus anterior muscle and a short one for the latissimus dorsi muscle (Fig 1D). Thus, it was considered as the LTA, as no similar artery was identified as separate branch of the AA (Fig 1D). Before ending its course, the AA gave a muscular branch for the latissimus dorsi which was considered as the "typical" TDA (Fig 1D). The thoracodorsal nerve coursed alone until the latissimus dorsi muscle, without accompanying the TDA, as usually. The rest arterial pattern was typical, with no further variations of the left brachial artery.

\section{DISCUSSION}

Variations of the AA branching pattern seem to be rather common. According to the classical anatomical textbooks [20], the typical pattern is observed in $65 \%$ of the cases, a LCCA arising from the BCT in $27 \%$, an AOA consisted of four branches in $2.5 \%$ and a right and left BCT in only $1.2 \%$. The rest of the cases presented a variety of arterial patterns (3.8\%).

During embryonic development, main vessels develop from a primary plexus of smaller capillaries. The prevailing conditions lead some vessels to enlarge and form definitive channels and other to regress. During this developmental phase, it is possible to appear different patterns in the vessels, including both the origin and/or course of either arteries or veins [17]. Normally, the AA derives from the embryonic phar- 

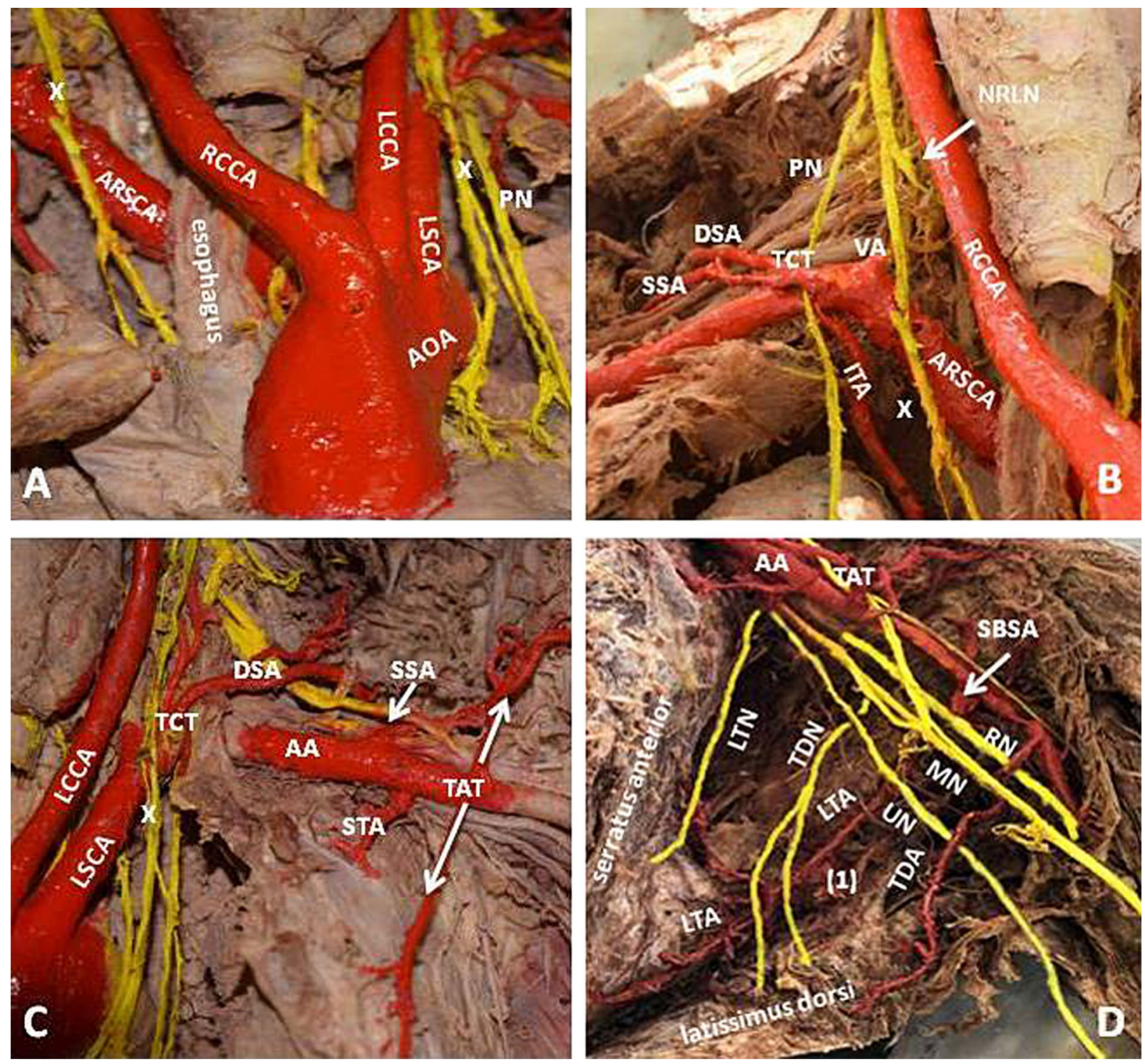

Figure 1. A. The aberrant right subclavian artery (ARSCA) arising from the posterior lateral aspect of the aortic arch (AOA) and passing posterior to the oesophagus. The right common carotid artery (RCCA), left common carotid artery (LCCA) and left subclavian artery (LSCA) originating from the AOA; B. The inferior laryngeal nerve arose approximately in the lower border of the thyroid cartilage and passed through the thyroid gland, instead of passing around the ARSCA, the so-called right non-recurrent laryngeal nerve (RNRLN). The internal thoracic artery (ITA) and the thyrocervical trunk (TCT) with the same point of origin; C. The suprascapsular artery (SSA) branching from the first part of the axillary artery (AA); D. The lateral thoracic artery (LTA) originates from the subscapural artery (SBSA) and gives rise to a muscular branch for the latissimus dorsi muscle (1). The thoracodorsal artery (TDA) arises independently from the $A A ; X$ — right vagus nerve; PN — phrenic nerve; VA — vertebral artery; TAT — thoracoacromial trunk; DSA — dorsal scapular artery; STA — superior thoracic artery; UN — ulnar nerve; $\mathrm{MN}$ — median nerve; TDN — thoracodorsal nerve; LTN — long thoracic nerve; RN — radian nerve.

yngeal arch arteries, namely from the aortic sac, the left dorsal aorta, and the left $4^{\text {th }} \mathrm{AOA}$ and its main branches, during the $5^{\text {th }}$ and $6^{\text {th }}$ weeks of gestation [10]. In cases of the ARSCA formation, the regression occurs instead between the $7^{\text {th }}$ intersegmental artery and the RCCA, so that the RSCA is then connected to the left dorsal aorta via the part of the right dorsal aorta which normally regresses. During growth, the
RSCA origin migrates until it is just distal to that of the LSCA $[1,10,16,21]$.

The variant $A O A$ types have a strong association with some congenital syndromes, such as DiGeorge syndrome, trisomy 13, 18 and 21, tetralogy of Fallot, origin of left coronary artery from the pulmonary artery, congenital polyvalvular disease, oesophageal atresia, and others [16]. 
In a meta-analysis [16], six types of the left-sided AOA concerning its branching pattern and course were identified:

$-1^{\text {st }}$ type - a normal pattern of three branches, BCT-LCCA-LSCA in $80.9 \%$;

$-2^{\text {nd }}$ type - the bovine arch, (common trunk of BCT and LCCA originating from the AOA followed by the LSCA) in $13.6 \%$;

- $3^{\text {rd }}$ type - the AOA of four branches (BCT-LCCALVA-LSCA) with an incidence of $2.9 \%$;

- $4^{\text {th }}$ type - a combination of bovine arch and aberrant LVA origin from the AOA in $0.4 \%$;

- $5^{\text {th }}$ type - an AOA which gave rise to the RSCA, followed by a bicarotid trunk (common trunk for RCCA and LCCA) and a LSCA, in $0.3 \%$;

- $6^{\text {th }}$ type - RCCA and LCCA from the AOA, followed by LSCA and ARSCA with an incidence of $0.7 \%$.

Other variants (left and right $B C T s$, right VA directly from the AOA, four branched AOA, double AOA, etc.) occur in less than $1 \%$.

Our case corresponds to type $6(0.7 \%)$. This type seems to have a low incidence in South American $(0.2 \%)$ and a high incidence in African populations (1.4\%) [16]. A median incidence was reported in European populations $(0.8 \%)$ [16]. In this type, the ARSCA follows usually a retro-oesophageal course $(80 \%$ of the cases) $[10,12,13,16,20]$ and rarely, the artery may pass between trachea and oesophagus (15\%) and more rarely in front of the trachea (5\%) [12]. Another important coexisted variant in such cases is the presence of a RNRLN almost totally (90-100\%) $[8,12,15,16]$. Occasionally, an aneurysm may be present at the ARSCA (12.8-14.9\%) [15]. Natsis et al. (2017) [14] reported the coexistence of an ARSCA with a thyroidea ima artery and a bilateral atypical internal thoracic artery origin in $17 \%$.

In most of the cases, the ARSCA seems to be asymptomatic. Nevertheless, the presence of dysphagia $(71.2 \%)$, dyspnoea $(18.7 \%)$, pain $(17.0 \%)$ or cough (7.6\%), due to oesophagus and trachea compression is not uncommon $[3,15,16]$. Some authors proposed a surgical correction, if these symptoms are rather annoying for the patient [7]. In children, the ARSCA variant may lead to recurrent respiratory infections due to compression on trachea [15]. The pressure on oesophagus may also cause a fistula and bleeding from the ARSCA $[5,16]$. A Kommerell diverticulum may occur at the point of the ARSCA emersion $[4,8,16]$.

Variant origins and courses of the SSA have been described. According to literature, the commonest variation is the direct origin of the SSA from the subclavian artery (10\%) [22], while the origins from the internal thoracic artery $(1-5.1 \%)$, from the costocervical trunk $(1 \%)$, or from the dorsal scapular artery $[10,19,22]$ are quite uncommon. The SSA origin from the $A A$, as in our case, is rather rare, with an incidence ranging between $1.6 \%$ [11] and 3.8\% [19]. The SSA supplies the proximal $4 / 5^{\text {ths }}$ of the clavicle and it is the exclusive blood supply for the middle one third of the clavicle [6]. This is important since $70-81 \%$ of the clavicular fractures occur in the middle third of the bone $[17,18]$. If the SSA originates from an atypical position, it is possible that a fractured clavicle may entrap its origin and course increasing the risk of damage. In our case, the SSA emerged from the $1^{\text {st }}$ part of the $A A$, just above the lateral third of the clavicle, which means that a middle clavicular fracture would not affect the artery [17].

Surgeries in the anterior neck and supraclavicular region to control the lymphogenous spread of head and neck cancer, may require the SSA ligation. Thus, knowledge of the possible variants in the origin and course of the artery are of paramount importance [9].

The incidence of anatomic variations of the major arteries of the upper extremities ranges from $11 \%$ to $24 \%$ [23]. The AA branches exhibit considerable variations [20]. According to the literature, the LTA originates from the $2^{\text {nd }}$ part of the $A A$ in $50 \%$, from the SBSA in $30 \%$ (as in our case), from the $1^{\text {st }}$ part of $A A$ in $11 \%$ and from the TAT in 7\% [22]. It even might be absent, in which case it is replaced by lateral perforating branches of the intercostal arteries or the TDA [22].

The formation of common trunks among the branches of AA is rather common, with the LTA forming a common trunk with the TDA in $10 \%$ and with SBSA in another 10\% [10]. Rarely, (3\%), the TDA might rise from the AA, separately from the SBSA [22]. In 4\% of the cases, the AA has been described to give rise to additional branches to the thoracic wall [10]. In the present study, the LTA emerges from the SBSA and provides also a branch for the latissimus dorsi muscle, while the TDA rises independently from the AA.

The abnormal course and origin of the AA branching pattern may cause confusion in angiographic studies and complicate a surgery [2].

\section{CONCLUSIONS}

In the current case, a retro-oesophageal ARSCA originating as a last branch of the AOA was detect- 
ed. This rare variation (less than 1\%) is accompanied by the following arterial variants: an axillary origin of the SSA (1.6-3.8\%), a LTA origin from the SBSA (30\%) and an independent origin of the left TDA (3\%). Combined arterial variants in the same individual are not unusual and are explained developmentally by the possible common embryonic origin of arterial variations. The occurrence of a variation may increase the possibility of coexistence of other arterial variants in the same region of the body. This fact must be kept in mind when a variant is encountered, either in cases where symptoms occur, or in cases of an accidental identification.

\section{REFERENCES}

1. Bhatia K, Ghabriel MN, Henneberg M. Anatomical variations in the branches of the human aortic arch: a recent study of a South Australian population. Folia Morphol. 2005; 64(3): 217-223, indexed in Pubmed: 16228958.

2. Cavdar S, Zeybek A, Bayramiçli M. Rare variation of the axillary artery. Clin Anat. 2000; 13(1): 66-68, doi: 10.1002/ (SICI)1098-2353(2000)13:1 <66::AID-CA8 > 3.0.CO;2-M, indexed in Pubmed: 10617889.

3. Celikyay ZR, Koner AE, Celikyay F, et al. Frequency and imaging findings of variations in human aortic arch anatomy based on multidetector computed tomography data. Clin Imaging. 2013; 37(6): 1011-1019, doi: 10.1016/j. clinimag.2013.07.008, indexed in Pubmed: 23938139.

4. Davidian M, Kee ST, Kato N, et al. Aneurysm of an aberrant right subclavian artery: treatment with PTFE covered stentgraft. J Vasc Surg. 1998; 28(2): 335-339, doi: 10.1016/ s0741-5214(98)70169-7, indexed in Pubmed: 9719329.

5. Feugier $P$, Lemoine $L$, Gruner $L$, et al. Arterioesophageal fistula: a rare complication of retroesophageal subclavian arteries. Ann Vasc Surg. 2003; 17(3): 302-305, doi: 10.1007/ s10016-001-0406-z, indexed in Pubmed: 12704542.

6. Havet E, Duparc F, Tobenas-Dujardin AC, et al. Vascular anatomical basis of clavicular non-union. Surg Radiol Anat. 2008; 30(1): 23-28, doi: 10.1007/s00276-007-0278-1, indexed in Pubmed: 18038111.

7. Karacan A, Türkvatan A, Karacan K. Anatomical variations of aortic arch branching: evaluation with computed tomographic angiography. Cardiol Young. 2014; 24(3): 485-493, doi: 10.1017/S1047951113000656, indexed in Pubmed: 23694814

8. Kobayashi M, Yuta A, Okamoto K, et al. Non-recurrent inferior laryngeal nerve with multiple arterial abnormalities. Acta Otolaryngol. 2007; 127(3): 332-336, doi: 10.1080/00016480600801415, indexed in Pubmed: 17364374.

9. Lamb DD. Radical neck dissection. Surg Technol. 2010: 449-454.

10. Lippert $H$, Pabst R. Arterialvariations in man. Classification and Frequency. Bergmann, Muenchen 1985.
11. Mishra S, Ajmani ML. Anomalous origin of suprascapular artery-a case report. J Anat Soc India. 2003; 52(2): 180-182.

12. Natsis K, Lazaridis N, Gkiouliava A, et al. Retro-oesophageal right subclavian artery in association with thyroid ima artery: a case report, clinical impact and review of the literature. Folia Morphol. 2016; 75(1): 130-135, doi: 10.5603/ FM.a2015.0080, indexed in Pubmed: 26383509.

13. Natsis KI, Tsitouridis IA, Didagelos MV, et al. Anatomical variations in the branches of the human aortic arch in 633 angiographies: clinical significance and literature review. Surg Radiol Anat. 2009; 31(5): 319-323, doi: 10.1007/s00276-008-0442-2, indexed in Pubmed: 19034377.

14. Natsis K, Didagelos M, Gkiouliava A, et al. The aberrant right subclavian artery: cadaveric study and literature review. Surg Radiol Anat. 2017; 39(5): 559-565, doi: 10.1007/s00276-016-1796-5, indexed in Pubmed: 27999944.

15. Polguj M, Chrzanowski $t$, Kasprzak JD, et al. The aberrant right subclavian artery (arteria lusoria): the morphological and clinical aspects of one of the most important variations--a systematic study of 141 reports. Scientific World J. 2014; 2014: 292734, doi: 10.1155/2014/292734, indexed in Pubmed: 25105156.

16. Popieluszko P, Henry BM, Sanna B, et al. A systematic review and meta-analysis of variations in branching patterns of the adult aortic arch. J Vasc Surg. 2018; 68(1): 298-306.e10, doi: 10.1016/j.jvs.2017.06.097, indexed in Pubmed: 28865978.

17. Pyrgakis P, Panagouli E, Venieratos D. Anomalous origin and course of the suprascapular artery combined with absence of the suprascapular vein: case study and clinical implications. N Am J Med Sci. 2013; 5(2): 129-133, doi: 10.4103/1947-2714.107534, indexed in Pubmed: 23641375.

18. Robinson CM. Fractures of the clavicle in the adult. Epidemiology and classification. J Bone Joint Surg Br. 1998; 80(3): 476-484, indexed in Pubmed: 9619941.

19. Singh R. Variations in the origin and course of the suprascapular artery: case report and literature review. J Vasc Bras. 2018; 17(1): 61-65, doi: 10.1590/16775449.008117, indexed in Pubmed: 29930683.

20. Standring, S.; Borley, N.R.; Collins, P. (eds.): Gray's anatomy. The anatomical basis of clinical practice. E Elsevier, Edinburgh 2008.

21. Tapia GP, Zhu X, Xu J, et al. Incidence of branching patterns variations of the arch in aortic dissection in Chinese patients. Medicine (Baltimore). 2015; 94(17): e795, doi: 10.1097/MD.0000000000000795, indexed in Pubmed: 25929931.

22. Tountas $C P$, Bergman RA. Anatomic variations of the upper extremity. Churchill Livingstone 1993.

23. Uglietta JP, Kadir S. Arteriographic study of variant arterial anatomy of the upper extremities. Cardiovasc Intervent Radiol. 1989; 12(3): 145-148, indexed in Pubmed: 2507150. 